\title{
WILEY-VCH
}

DOI: 10.1002/ ((please add manuscript number))

Article type: Full Paper

\section{Surface mediated alignment of long pitch chiral nematic liquid crystal structures}

Inge Nys, Jeroen Beeckman*, Kristiaan Neyts

Inge Nys, Prof. Jeroen Beeckman, Prof. Kristiaan Neyts

Technologiepark Zwijnaarde 15, Ghent, 9052, Belgium

Department of Electronics and Information Systems, Ghent University

E-mail: jeroen.beeckman@ugent.be

Keywords: liquid crystals, surface topography, periodic anchoring, directional growth, cholesteric fingers

Chiral nematic liquid crystals are stimuli-responsive soft materials that can self-organize into complex chiral superstructures. Control and manipulation of the chiral ordering is essential in the development of functional electro-optic devices. Here, the effect of surface topography is combined with an applied electric field to steer the directional growth of cholesteric fingers in a cell with thickness larger than the chiral pitch. The periodic e-beam resist grating gives rise to micrometer-scale periodic variations of the surface anchoring. By applying a voltage to the cell, controlled growth of cholesteric fingers along the surface grating is observed. The 2D liquid crystal director configuration at different voltages is identified by numerical simulations based on finite element Q-tensor theory. The results demonstrate that samples with modulated surface chemistry lead to enhanced control over the formation of chiral structures. This can stimulate the development of electro-optic devices (diffraction gratings, lasers, displays, etc.) with improved characteristics.

\section{Introduction}

Chiral nematic liquid crystals (CLCs) are stimuli-responsive soft materials that can selforganize into hierarchical superstructures. CLC in bulk spontaneously forms periodic helical structures and the distance over which the molecular director rotates by $2 \pi$ is called the pitch 


\section{WILEY-VCH}

p. This makes CLCs interesting for the use in self-assembled and tunable lasers, reflectors, diffraction gratings and many more ${ }^{[1]}$ The stimuli-responsiveness of CLCs can lead to controllable manipulation of the director configuration and can be exploited to dynamically reconfigure the helical superstructures. ${ }^{[2]}$

The director configuration in a confined geometry depends on a delicate balance between surface anchoring, elastic energy and, if an electric field is applied, dielectric energy. Changing this equilibrium allows to obtain novel superstructures. Being able to control and influence the alignment of LC is therefore of primordial importance, both from a fundamental and an application-oriented point of view. Herein, we apply surface topography to steer the directional growth of long pitch $(p=4.6 \mu \mathrm{m})$ CLC structures. We demonstrate that thin stripes $(200 \mathrm{~nm})$ of e-beam resist on the surface can dramatically influence the formation and growth of chiral superstructures. The director configuration is further affected by applying a voltage to the cell. When the voltage is decreased below the dielectric unwinding threshold, growth of cholesteric fingers (CFs) along the surface gratings is observed.

In cells with uniform homeotropic alignment filled with CLC, four types of CFs have been reported in the past and their behavior under influence of an applied electric field was studied. ${ }^{[3]}$ The director profile in these CFs has been simulated and experimentally verified with fluorescence confocal polarizing microscopy. ${ }^{[3]}$ To differentiate between the different types of CFs we will use the classification of Oswald et al. ${ }^{[3 d]}$ In the cells with surface gratings that we investigate here, the experimental results suggest a link with the cholesteric finger of the first kind (CF1) observed in homeotropically aligned cells. The CF1 can continuously form from the translationally invariant configuration and easily nucleate from the homeotropic unwound structure, which explains their abundance. The CF1 does not contain dislincations and the director roughly makes a $2 \pi$ twist around an axis that is tilted away from the cell normal. ${ }^{[3]}$ The differences and similarities between the $\mathrm{CF} 1$ and the $\mathrm{CF}$ observed in our cells will be studied by finite element (FE) simulations and discussed. 


\section{WILEY-VCH}

In the past, the effect of surface topography on the alignment of nematic liquid crystal (NLC) has been studied. Surface structures were made by nano-imprint lithography, soft embossing, replica moulding, laser scanning lithography, ion beam milling, nanosphere lithography and e-beam lithography. ${ }^{[4]}$ As explained by Berreman's groove theory, NLC molecules preferentially align along the groove direction and not perpendicular to it since there is an elastic energy penalty associated with the alignment perpendicular to the grooves. ${ }^{[5]}$ The smaller the period of the surface structure and the deeper the trenches, the stronger the tendency to align along the stripes. The resulting NLC alignment close to the surface depends on the combined effect of surface topography and surface chemistry, related to the polymer backbone of the alignment material. ${ }^{[4 c]}$ When NLC is replaced by CLC the situation becomes more complicated and Berreman's groove theory is not simply applicable since uniform alignment deviates from the elastic energy minimum. In this case, besides the surface topography and surface chemistry, also the chiral pitch and the cell thickness define the resulting director configuration. Outram et al. exploited the effect of surface topography by creating a mold-templated surface to align a short pitch $(p=320 \mathrm{~nm})$ uniform lying helix while Carbone et al. used a surface produced by two-photon excitation laser-lithography to break the azimuthal degeneracy of the homeotropic alignment layer and roughly align a long

pitch $(p=4 \mu \mathrm{m})$ uniform lying helix. ${ }^{[4 \mathrm{~d}, 4 \mathrm{e}]}$ In these cases, the whole surface structure consisted of the same material and the effect on the CLC alignment was solely related to the surface topography. In our experiments, we create a periodic grating of stripes of e-beam resist, separated by stripes where the resist is removed. This results in a surface structure where the LC is in contact with the e-beam resist in some regions and with the ITO-coated glass in other regions. The surface chemistry of both materials is different and this results in periodically varying anchoring: homeotropic above the ITO stripes and planar degenerate above the ebeam resist stripes. The experiments and simulations indicate that this effect is more important than the effect of surface topography in our samples. 


\section{WILEY-VCH}

The e-beam lithography method that we propose can be an alternative for existing techniques that allow micrometer-scale periodic variation of the anchoring. ${ }^{[6]}$ Self-assembled monolayers were used in the past to obtain patterned homeotropic and planar alignment and two different photo-alignment techniques were established. ${ }^{[6 \mathrm{c}, 6 \mathrm{f}, 6 \mathrm{~g}]}$ Alternatively, a polymerized CLC layer was tested as a periodic alignment layer and a polyimide layer with planar alignment was coated with a chromium complex surfactant for homeotropic anchoring and patterned by

hard-contact photolithography. ${ }^{[6 a, 6 b]}$ Our straight-forward lithography technique has the advantage that small scale structures with different shapes are easily achievable and that the effect of surface topography can be combined with periodically modulated surface chemistry. Different thicknesses of the resist layer and thinner cell gaps can be used in the future to further exploit the influence of surface topography. Depending on the lateral dimensions that are envisioned, e-beam lithography can also be replaced by conventional UV lithography.

\section{Experimental results}

A grating structure is formed on the bottom substrate of the cell by periodic stripes of e-beam resist (Figure 1). As described in the experimental section, $12 \mu \mathrm{m}$ gap cells are formed with one patterned and one flat glass substrate and filled with CLC $(p=4.6 \mu \mathrm{m})$. Different periodicities $\Lambda$ of the surface grating were tested, corresponding to $\mathrm{p} / 4, \mathrm{p} / 2, \mathrm{p}$ and $2 \mathrm{p}$. Moreover, the periodicity was slightly varied (+/-15\%) with respect to the equilibrium pitch of the CLC.

At high E fields, the polarizing optical microscopy (POM) images using crossed polarizers look dark (Figure 1c), indicating vertical LC alignment in the bulk. When the voltage is decreased, a randomly oriented cholesteric structure appears in the regions with unpatterned bottom substrate while the vertical alignment is initially conserved in the patterned area (Figure 1d). A slight further decrease of the voltage leads to directional growth of bright 


\section{WILEY-VCH}

stripes, so-called cholesteric fingers ( $\mathrm{CFs})$, parallel to the e-beam resist stripes (Figure 1e). More CFs start to grow and fill the entire grating area when the voltage is further decreased (figure 1f). Perturbations of the directional growth appear at small voltages (figure 1g-h). The described behavior, with growth of CFs along the grating lines at intermediate voltages, is only observed when the surface periodicity $\Lambda$ of the grating approximately equals the pitch of the CLC. Surface periodicities close to $\mathrm{p} / 4, \mathrm{p} / 2$ and $2 \mathrm{p}$ did not give rise to directional growth of chiral structures (Figure 2). This indicates that the ratio between the surface periodicity and the pitch of the material is crucial. On the other hand, a small deviation (up to $15 \%$ ) between both did not remarkably change the results (see Figure 2).

By carefully analyzing the POM images of the grating area at high voltages (Figure 3) we can conclude that the grating introduces a periodic alignment at the surface. The uniform top surface is homeotropically aligned and the non-patterned e-beam resist layer gives rise to planar degenerate anchoring. Inside the grating area, periodic stripes of homeotropic and planar degenerate anchoring are observed (Figure 3). An additional cell was fabricated with some isolated stripes of resist removed to clearly distinguish the areas with and without ebeam resist (Figure 3a). The POM images confirm that the ITO covered glass in the grating is responsible for the stripes with homeotropic alignment while the remaining stripes of e-beam resist give rise to planar degenerate anchoring.

To further illustrate the dynamic growth of the CFs in our grating structure, two movies are incorporated in the supporting information. The first movie shows that the nucleation and growth of CFs starts at the edges of the grating and illustrates the interaction of the CFs propagating in the opposite direction. In the second movie the behavior of the CFs at lower voltages can be studied. Two snapshots from the first movie are shown in Figure 4 to indicate different types of interactions between CFs.

\section{FE Q-tensor simulations}




\section{WILEY-VCH}

To identify the director configuration in the observed CFs, finite element Q-tensor simulations were performed. The Jones calculus (combined for red $\lambda=620 \mathrm{~nm}$, green $\lambda=520 \mathrm{~nm}$ and blue $\lambda=420 \mathrm{~nm}$ ) is applied to find the general behavior of the transmission between crossed polarizers. We limit ourselves to the simulation of a 2D cross section (in the xz plane) of the CFs since this mostly suffices to understand the experimentally observed behavior. Effects in the third dimension (along y), such as the interaction of the finger tips and in-plane buckling of the fingers, are discussed separately. The Q-tensor, which is a symmetric, traceless order tensor of rank two, is used in the simulations to represent the local director and the order parameter in the LC. The Landau-de Gennes free energy, containing a thermotropic bulk term, an elastic distortion term, an electrostatic energy term and a surface term, is minimized to find the director configuration. The elastic and dielectric properties of E7 are used in the simulations $\left(\mathrm{K} 11=11.1 \mathrm{pN}, \mathrm{K} 22=6.5 \mathrm{pN}, \mathrm{K} 33=17.1 \mathrm{pN}, \varepsilon_{\perp}=5.2, \varepsilon_{/ /}=19\right)$ together with some characteristic values for the bulk thermotropic coefficients. Very few measurements of thermotropic coefficients are found in the literature so we use thermotropic coefficients based on the ones measured for $5 \mathrm{CB} .{ }^{[7]}$ To increase the natural length scale of variations in the order parameters and to obtain faster numerical convergence we use 10 times smaller values for the thermotropic coefficients. ${ }^{[7 \mathrm{~b}-\mathrm{d}]}$ More details of the simulation method can be found in the supporting information and in previous articles. ${ }^{[1 \mathrm{~d}, 6 \mathrm{~g}]}$

For the simulations of our cells with a surface grating, the initial director configuration is inspired by the CF1 in homeotropic cells and experimental observations are taken into account to define the boundary conditions. We used a patterned bottom substrate with stripes of planar (on the e-beam resist) and homeotropic (on ITO) alignment. The other boundary conditions are homeotropic alignment at the top and periodic boundary conditions (identical Q-tensor at left and right interface). The azimuthal anchoring strength was assumed to be weak $\left(10^{-5} \mathrm{~J} / \mathrm{m}^{2}\right)$ to represent the experimentally observed planar degenerate anchoring, while the polar anchoring strength was set to $10^{-3} \mathrm{~J} / \mathrm{m}^{2}$, corresponding to strong anchoring. The easy 


\section{WILEY-VCH}

axis for the planar alignment was chosen along the xy-diagonal, but this has a minor influence on the results since the azimuthal anchoring strength is weak. The physical height difference originating from the resist stripes $(200 \mathrm{~nm})$ is not taken into account since it is negligible with respect to the other relevant length scales $(d=12 \mu \mathrm{m}, \mathrm{p}=4.6 \mu \mathrm{m})$. In the experiments not every grating line is filled with a CF, as the CFs are typically surrounded by a broader homeotropic unwound area. For this reason, we made the simulation domain a bit wider than the surface grating period $\Lambda$ (width $6.6 \mu \mathrm{m}$ instead of $\Lambda=4.6 \mu \mathrm{m}$ ), by increasing the width of the homeotropic areas while preserving the width of the planar region $(2.3 \mu \mathrm{m})$. This width has only a minor influence on the results as is demonstrated in the supporting information

\section{(Figure S1).}

Simulation results for the CF1 that appears in a device with pure homeotropic anchoring at different voltages are illustrated in Figure 5, where the director configuration is shown together with the transmission between crossed polarizers and a diagram for the elastic distortion energy and dielectric energy. For ease of comparison, we use the same cell thickness $(\mathrm{d}=12 \mu \mathrm{m})$, material pitch $(\mathrm{p}=4.6 \mu \mathrm{m})$ and width of the simulation area $(6.6 \mu \mathrm{m})$ as in the simulations for the patterned cell.

The simulation results in our cell with a periodic grating at the bottom substrate are summarized in Figure 6 for different voltages. Both the director configuration, the electric and elastic energy distribution and the transmission between crossed polarizers are shown. Strong polar anchoring with an abrupt change between the planar and homeotropic alignment regions is assumed. This leads to high values in the simulated distortion energy close to the edges between both regions (Figure 6).

\section{Discussion}

At high E field, the cholesteric structure is completely unwound and the director is aligned vertically in the bulk (see Figure 1c). This state is compatible with the homeotropic alignment 


\section{WILEY-VCH}

on the top substrate and minimizes the dielectric energy. A decrease of the voltage leads to directional growth of bright $\mathrm{CFs}$ along the grating area (Figure 1e). We experimentally observe that the CFs consist of two bright edges separated by a darker middle area. This hints that we are dealing with CFs that accommodate a $2 \pi$ twist (contrary to the CF3 that accommodates a $\pi$ twist) and explains why directional growth of CFs along the grating area is only observed for $\Lambda \approx \mathrm{p}$ and not for $\Lambda \approx \mathrm{p} / 2, \mathrm{p} / 4$ or $2 \mathrm{p}$. The width of the planar alignment region should be close to $\mathrm{p} / 2$ to achieve a CF that accommodates a $2 \pi$ twist with low elastic energy. The absence of a strong asymmetry in the CFs suggests a link with the CF1 observed in homeotropically aligned cells. Moreover, the CFs that grow inside the grating area are a continuation of CFs that are present in the non-patterned area, where CF1 is known to be by far the most abundant. ${ }^{[3]}$

At high voltages (above $5 \mathrm{~V}$ ) the $\mathrm{CF} 1$ is unstable and unwinds to the homeotropic state while at voltages slightly below the dielectric unwinding threshold a CF1 is formed with the helical twist axis parallel to the glass substrates (Figure 5). When the voltage is further decreased, the contribution of the dielectric energy becomes smaller and the CF1 becomes wider to make the variation in the twist more sinusoidal. At low voltages, the helical twist axis tilts out of the plane to minimize the elastic energy (Figure 5). This agrees with a smooth transition from individual CFs separated by narrow homeotropic stripes towards CFs that merge and finally a (modulated) translationally invariant configuration with a (close to) vertical twist axis. ${ }^{[3,8]}$ Our experimental results show strong similarities to this behavior. At high voltages, CFs typically start growing from the edges of the grating area but sometimes they also nucleate at defects that are present. The CFs are centered on top of the planar alignment region (see Figure 3d), in agreement with the simulations (Figures 6). When the voltage is decreased, the CFs become wider and the color observed in POM changes because another retardation is experienced by light propagating through the cell. The simulated director structure (for all different voltages) strongly resembles the CF1 in cells with pure homeotropic alignment, 


\section{WILEY-VCH}

although in our experiments the CFs are anchored to the patterned bottom substrate (Figure 6).

The CF1 was previously described as a quadrupolar defect cluster of half-integer disclinations

with opposite signs $s=+-1 / 2 .^{[3 \mathrm{a}, 3 \mathrm{e}]}$ In our cells the nonsingular $\lambda^{-1 / 2}$ and $\lambda^{+1 / 2}$ defect lines close

to the homeotropic substrate are conserved but at the bottom substrate the $\lambda^{+1 / 2}$ defect is modified and the $\lambda^{-1 / 2}$ is removed. The anchoring at the bottom substrate hinders the motion of the CF (in the $\mathrm{x}$ and $\mathrm{z}$ direction) and fixes the orientation of the CFs in space. In other words, the surface grating structure dictates the orientation of the CFs, explaining the experimentally observed directional growth. Similar to the CF1 in homeotropic cells, the CFs in our cells are broadening and the twist axis is tilted towards the surface normal at low voltages to decrease the elastic energy (Figure 6). This agrees with the experimental observations where a broadening of the finger and a change in transmission are observed (Figure 1g-h).

Comparison of the simulated results for the transmission between crossed polarizers (Figure 6) with the experimental POM images (Figure 1) shows a fairly good agreement. The Jones calculus is not ideally suited to simulate the transmission in thick cells (high retardation leads to strong wavelength dependency) and structures with small lateral dimensions (due to diffraction effects), but can uncover the general behavior. Both experimentally and in the simulations, at high voltages the CFs appear symmetric in transmission. The amount of transmission for different orientations of the CF between crossed polarizers can be evaluated experimentally comparing the brightness of the $\mathrm{CF}$ with respect to the background in Figure 1 (e) and (i). At high voltages, both at $0^{\circ}$ and $45^{\circ}$ between crossed polarizers, the edges of the finger are brighter than the middle and the transmission is higher when the $\mathrm{CF}$ is oriented at $45^{\circ}$ between crossed polarizers. At small voltages, a more colorful POM image is observed (Figure 1). These observations are in agreement with the simulations. At small voltages we observe experimentally that perturbations of the directional growth appear and manifest themselves by deviations of the CFs away from the direction dictated by 


\section{WILEY-VCH}

the surface grating (figure 1g-h). The FE Q-tensor simulations only describe the director configuration in a 2D cross section of the $\mathrm{CF}$ but deviations in the third dimension can be explained phenomenologically. Even though the voltage is decreased in small steps, the grating area does not completely fill with parallel CFs, and some homeotropic unwound areas are conserved (figure 1f). These unwound areas are less stable at lower voltages and to compensate for this, the region can be filled with new cholesteric structures, or the already present CFs can start to buckle in the plane of the cell. In our experiments, the anchoring of the CFs to the bottom substrate is not strong enough to prevent in-plane buckling and this mechanism dominates over nucleation and growth of new CFs at low voltages (Figure 1g, Movie S2). We expect that this is related to the $\mathrm{d} / \mathrm{p}$ ratio, as in-plane buckling seems move favorable for cells with a large $\mathrm{d} / \mathrm{p}$ ratio and weak surface anchoring.

Experimentally we see that at high voltages the fingers do not always keep growing on a straight line, but sometimes shift laterally (along $\mathrm{x}$ ) to one of the next grating lines to continue their growth (Figure 1). This deviation from a straight line can be related to surface imperfections or can be initiated by the repulsion between two fingers growing in opposite directions (Figure 1, Figure 4 and movie S1 in supporting information). The fact that the fingers deviate relatively easily from the structure can also indicate that the pinning at the surface is relatively weak. We expect that these deviations could be reduced by increasing the height of the surface perturbation or by making the cell thinner, to increase the relative importance of the surface energy. The structural imperfections could also be reduced by optimizing the e-beam process: resist reflow, resist development at decreased temperatures and (partial) development with o-xylene are known to diminish the surface roughness. ${ }^{[9]}$ Finally the interaction of finger tips in the third dimension was experimentally studied (Figure 4 and movieS1 in supporting information) and compared to the behavior of known CFs in homeotropic cells. As already identified by Ribiere et al. in the early 90's, the two tips at opposite ends of a finite segment of a CF1 (in a homeotropic cell) are not identical since there 


\section{WILEY-VCH}

is no mirror symmetry in a CLC. ${ }^{[3 b, 8]}$ At the end with the lower energy (called the normal tip) the twist has the same sign as the spontaneous twist of the free CLC, while at the other end (called the abnormal tip) the twist is mixed and the total energy is higher. ${ }^{[3 b, 8]}$ Normal tips appear rounded while abnormal tips appear sharper. Two tips of the same sign repel each other while tips of opposite sign collapse together. Moreover, an abnormal tip can collapse with the side of a finger and form a T-branch while a normal tip is repelled by the side of a finger. All these effects can also be identified in our experiments. At the edges of the e-beam resist stripes, typically fingers with normal tips start growing along the grating lines, as is energetically most favorable (Figure 4). Two fingers that are growing from the opposite sides repel each other and never merge. Moreover, if one of the fingers impinges on the side of another finger, no connection is formed (Figure 4). We can also identify a few fingers with abnormal tips that grow into the grating structure. When they collide with the side of another finger they can indeed merge and form a T-branch, illustrating the abnormal character of these tips corresponding with an opposite sign of twist (Figure 4).

\section{Conclusion}

To summarize, we have combined a long pitch $(\mathrm{p}=4.6 \mu \mathrm{m})$ CLC with a $200 \mathrm{~nm}$ thick surface grating created by e-beam lithography to study the directional growth of CFs as a function of the voltage. The surface structure leads to periodic stripes with homeotropic anchoring on the ITO and planar degenerate anchoring on the e-beam resist. The surface grating allows to control the formation and growth of CFs in relatively thick cells, that would otherwise lead to randomly oriented CLC structures. ${ }^{[3 \mathrm{~d}]}$ New supramolecular chiral structures, related to the known CF1 in homeotropically aligned cells, are identified and studied by FE Q-tensor simulations. A detailed analysis of the elastic distortion energy and the electric energy for different voltages is given and the transmission between crossed polarizers is simulated with the Jones calculus. Our straight-forward lithography technique has the advantage that also 


\section{WILEY-VCH}

complex patterns can be defined on a small scale. The combined effect of structured anchoring (planar or homeotropic) and the topography of the patterned areas, can be further exploited to manipulate the LC director configuration, for example to realize in-plane lying helical structures. This versatile method for patterning LC anchoring, that may be combined with appropriately patterned electrodes, can be interesting for application in different electrooptical devices such as diffraction gratings, lasers, geometrical phase optics and displays.

\section{Experimental Section}

Sample Preparation: A $200 \mathrm{~nm}$ layer of positive e-beam resist AR-P 6200.09 (CSAR 62) is spincoated on top of the ITO covered glass substrates (Delta Technologies, ITO coated float glass $1.1 \mathrm{~mm}$ ) at $4000 \mathrm{rpm}$ for $60 \mathrm{~s}$. The layer is tempered at $150^{\circ} \mathrm{C}$ for $1 \mathrm{~min}$ on a hot plate and the resist is exposed with the Voyager e-beam system (Raith). The low current (LC) mode with $60 \mu \mathrm{m}$ aperture was used and the exposure dose was $85 \mu \mathrm{C} / \mathrm{cm}^{2}$. Development: $1 \mathrm{~min} \mathrm{n}$ amyl acetate, stopping: $30 \mathrm{~s}$ IPA and $30 \mathrm{~s}$ DI water.

The dimension of the patterned areas is $500 \mu \mathrm{m} \times 500 \mu \mathrm{m}$ and different gratings are combined on the same substrate. To make a LC cell, one patterned substrate is glued together with a flat ITO covered glass substrate (Delta Technologies, ITO coated float glass $1.1 \mathrm{~mm}$ ). The flat glass substrates were treated with UV-ozone $\left(15 \mathrm{~min}\right.$ at $90^{\circ} \mathrm{C}$, Novascan) and covered with a homeotropic alignment layer (SE4811, Nissan Chemical Industries). Spin speed 2500rpm, spin time $15 \mathrm{~s}$, tempered $2 \mathrm{~min}$ at $90^{\circ}$ on the hot plate, baked $1 \mathrm{~h}$ at $180^{\circ}$ in the oven. The substrates were glued together using a glue (NOA68) with spherical spacer balls (12 $\mu \mathrm{m})$ near the edges. A mixture of the NLC E7 and the chiral dopant BDH 1305 was used in the experiments. The pitch was measured to be $\mathrm{p}=4.6 \mu \mathrm{m}$ with the Grandjean-Cano wedge method. The cell, with cell gap $\mathrm{d}=12 \mu \mathrm{m}$, is filled above the isotropic-nematic transition temperature and cooled down slowly to room-temperature.

\section{Supporting Information}




\section{WILEY-VCH}

Supporting Information is available from the Wiley Online Library or from the author.

\section{Acknowledgements}

The authors would like to acknowledge funding by the Research Foundation - Flanders (FWO) in the form of a PhD Fellowship grant for I. Nys. The authors would like to acknowledge the funding of the e-beam by Hercules (research infrastructure AUGE/13/19).

Received: ((will be filled in by the editorial staff))

Revised: ((will be filled in by the editorial staff)) Published online: ((will be filled in by the editorial staff))

\section{References}

[1] a) W. Hu, H. Zhao, L. Song, Z. Yang, H. Cao, Z. Cheng, Q. Liu, H. Yang, Adv. Mat.

2010, 22, 468; b) H. Coles, S. Morris, Nat. Photonics 2010, 4, 676; c) J. Beeckman, K. Neyts, P.J.M. Vanbrabant, Opt. Eng. 2011, 50, 081202; d) I. Nys, J. Beeckman, K. Neyts, Soft Matter 2015, 11, 7802; e) Z.-G. Zheng, Y. Li, H. K. Bisoyi, L. Wang, T. J. Bunning, Q. Li, Nature 2016, 531, 352; f) J. Kobashi, H. Yoshida, M. Ozaki, Nat. Photonics 2016, 10, 389; g)

D. Zhao, H. He, X. Gu, L. Guo, K. S. Wong, J. W. Y. Lam, B. Z. Tang, Adv. Opt. Mater. 2016, 4, 534 .

[2] a) L. Zhang, L. Wang, U. S. Hiremath, H. K. Bisoyi, G. G. Nair, C. V. Yelamaggad, A. M. Urbas, T. J. Bunning, Q. Li, Adv. Mater. 2017, 29, 1700676; b) Z.-G. Zheng, R. S. Zola, H. K. Bisoyi, L. Wang, Y. Li, T. J. Bunning, Q. Li, Adv. Mater. 2017, 29, 1701903; c) H.-C. Jau, Y. Li, C.-C. Li, C.-W. Chen, C.-T. Wang, H. K. Bisoyi, T.-H. Lin, T. J. Bunning, Q. Li, Adv. Opt. Mater. 2015, 3, 166; d) L. Wang, Q. Li, Adv. Funct. Mater. 2016, 26, 10-28.

[3] a) I. Smalyukh, B. Senyuk, P. Palffy-Muhoray, O. D. Lavrentovich, H. Huang, E. C. Gartland, V. H. Bodnar, T. Kosa, B. Taheri, Phys. Rev. E 2005, 72, 061707;b) J. Baudry, S. Pirkl, P. Oswald, Phys. Rev. E 1998, 57, 3038; c) L. Gil, J.M. Gilli, Phys. Rev. Lett. 1998, 80, 5742; d) P. Oswald, J. Baudry, S. Pirkl, Phys. Rep. 2000, 337, 67; e) P. J. Ackerman, Z. Qi, Y. Lin, C. W. Twombly, M. J. Laviada, Y. Lansac, I. I. Smalyukh, Sci. Rep. 2012, 2, 414; f) J. Baudry, S. Pirkl, P. Oswald, Phys. Rev. E 1999, 59, 5562; g) S. Pirkl, P. Oswald, Liq. Cryst. 


\section{WILEY-VCH}

2001, 28, 299; h) O. S. Tarasov, A. P. Krekhov, L. Kramer, Phys. Rev. E 2003, 68, 031708; i)

P. Oswald, A. Dequidt, Phys. Rev. E 2008, 77, 051706; i) S. Pirkl, Cryst. Res. Technol. 2009, 44, 1323; j) Y. Guo, S. Afghah, J. Xiang, O. D. Lavrentovich, R. L. B. Selinger, Q.-H. Wei, Soft Matter 2016, 12, 6312; k) P. M. Rupnik, D. Lisjak, M. Copic, S. Copar, A. Mertelj, Sci. $A d v . \mathbf{2 0 1 7}, 3, \mathrm{e} 1701336$.

[4] a) H. Takahashi, T.Sakamoto, H. Okada , J. Appl. Phys. 2010, 108, 113529; b) H.-G. Park, J.-J. Lee, K.-Y. Dong, B.-Y. Oh, Y.-H. Kim, H.-Y. Jeong, B.-K. Ju, D.-S.Seo, Soft Matter 2011, 7, 5610; c) D.-R. Chiou, L.-J. Chen, Langmuir 2006, 22, 9403-9408; d) B. I.

Outram, S. J. Elston, R. Tuffin, S. Siemianowski, B. Snow, J. Appl. Phys. 2013, 113, 213111;

e) G. Carbone, D. Corbett, S. J. Elston, P. Raynes, A. Jesacher, R. Simmonds, M. Booth, Mol. Cryst. Liq. Cryst. 2011, 544, 37-49; f) S. P. Palto, A. R. Geivandov, I. V. Kasyanova, V. V. Artemov, M. V. Gorkunov, JETP Letters 2017, 105, 174-178; g) M. Wahle, K. Brassat, J. Ebel, J. Bürger, J. K. N. Linder, H.-S. Kitzerow, Opt. Express 2017, 25, 22608; h) T. Kagajyo, K. Fujibayashi, T. Shimamura, H. Okada, H. Onnagawa, Jpn. J. Appl. Phys. 2005, $44,578-581$.

[5] D. W. Berreman, Mol. Cryst. Liq. Cryst. 1973, 23, 215.

[6] a) L. Komitov, G. P. Bryan-Brown, E. L. Wood, A. B. J. Smout, J. Appl. Phys. 1999, 86, 3508; b) G. Hegde, L. Komitov, Appl. Phys. Lett. 2010, 96, 113503; c) B.-W. Lee, N. A. Clark, Science 2001, 291, 2576-2580; d) V. K. Gupta, N. L. Abbott, Science 1997, 276, 1533; e) S. Park, C. Padeste, H. Schift, J. Gobrecht, T. Scharf, Adv. Mater. 2005, 17, 1398; f) W.-Z. Chen, Y.-T. Tsai, T.-H. Lin, Appl. Phys. Lett. 2009, 94, 201114; g) I. Nys, K. Chen, J.

Beeckman, K. Neyts, DOI: 10.1002/adom.201701163 h) X. Li, J. C. Armas-Perez, J. A. Martinez-Gonzalez, X.Liu, H. Xie, C. Bishop, J. P. Hernandez-Ortiz, R. Zhang, J. J. de Pablo, P. F. Nealey, Soft Matter 2016, 12, 8595; i) J. A. Martinez-Gonzalez, X. Li, M. Sadati, Y. Zhou, R. Zhang, P. F. Nealey, J. J. de Pablo, Nat. Comm. 2017, 8, 15854. 


\section{WILEY-VCH}

[7] a) H. J. Coles, Mol. Cryst. Liq. Cryst. 1978, 49, 67; b) L. A. Parry-Jones, R. B. Meyer,

S. J. Elston, J. Appl. Phys. 2009, 106, 014510; c) E. Willman, F. A. Fernández, R. James, S.

E. Day, J. Disp. Technol. 2008, 4, 276; d) N. J. Mottram, C. M. Care, D. J. Cleaver, Phys. Rev. E 2006, 74, 041703 .

[8] a) P. Ribiere, P. Oswald, J. Phys. France 1990, 51, 1703; b) P. Oswald, J. Baudry, T.

Rondepierre, Phys. Rev. E 2004, 70, 041702.

[9] S. Thoms, D. S. Macintyre, J. Vac. Sci. Technol., B. 2014, 32, 06 FJ01. 


\section{WILEY-VCH}

(a)
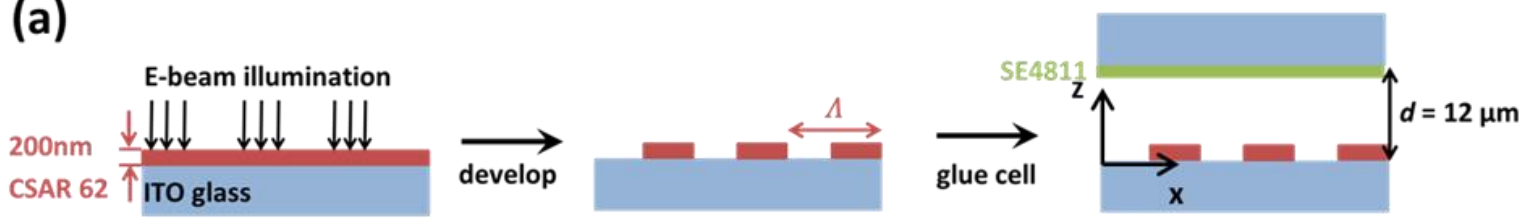

(b)

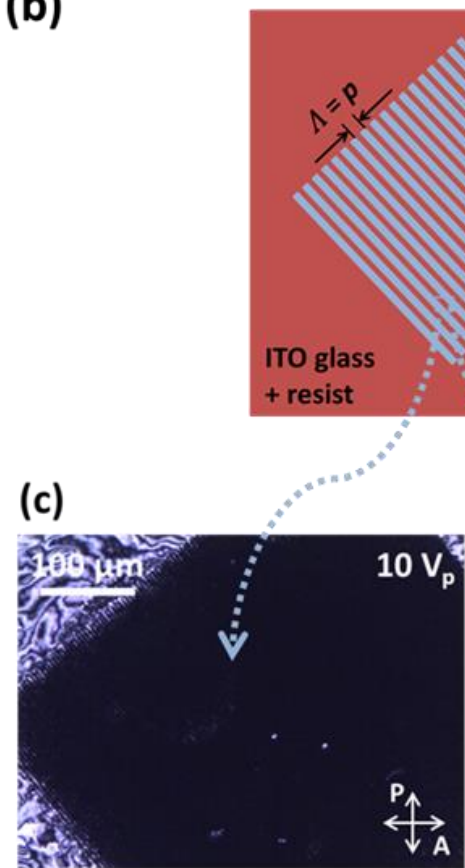

(e)

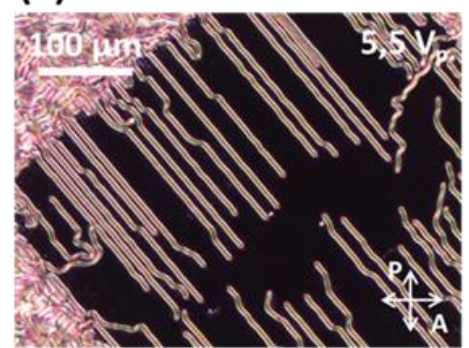

(g)

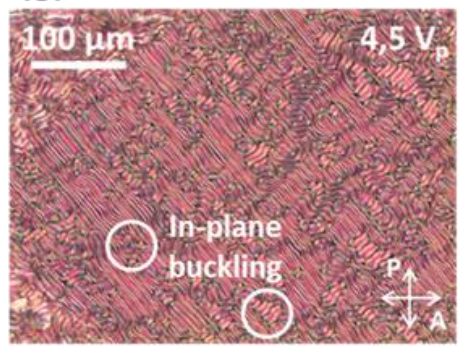

(d) ${ }^{n}$

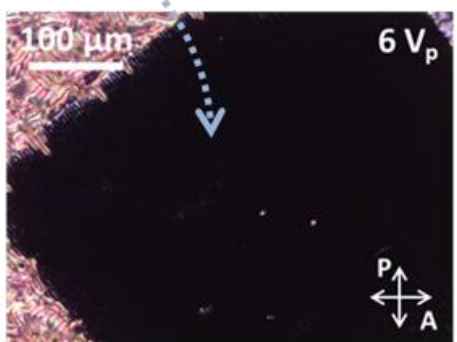

(f)

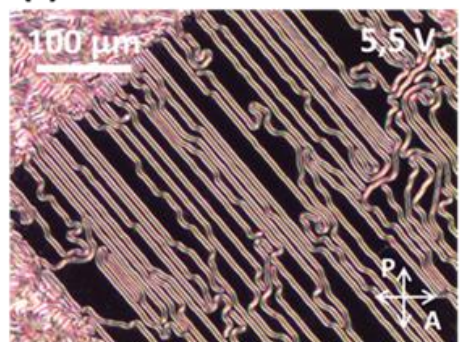

(h)

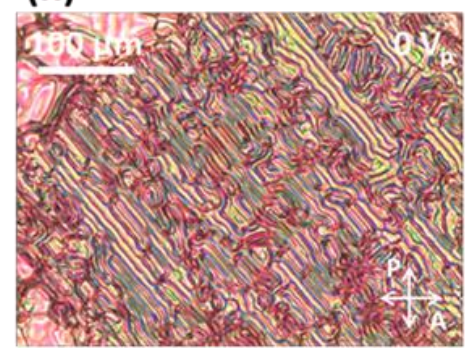

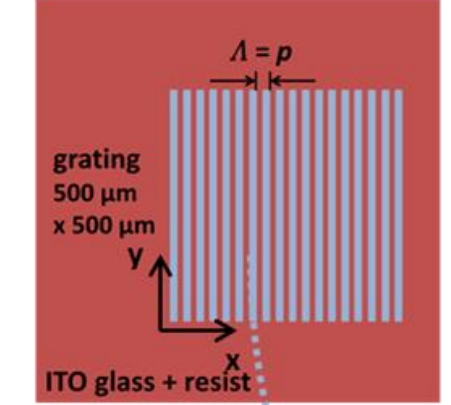

(i)

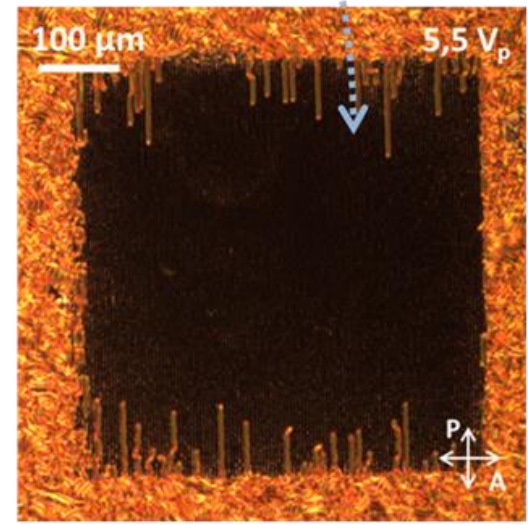

(j)

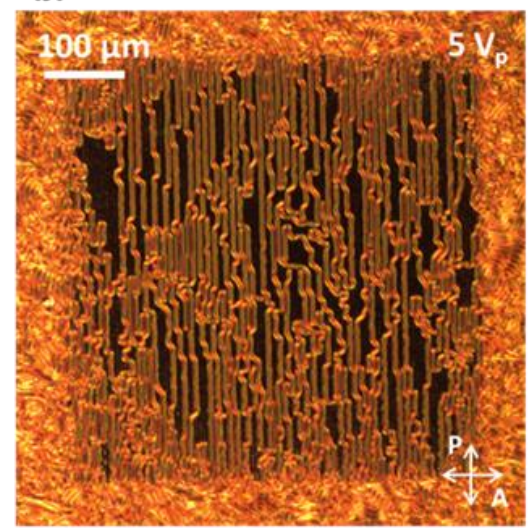

Figure 1. a) overview of the production process (photoresist CSAR 62 in red); b) schematic representation of the grating area and period $\Lambda ; \mathrm{c}-\mathrm{h}$ ) and $\mathrm{i}-\mathrm{j}$ ) POM images for decreasing voltages, with the grating rotated over $45^{\circ}(\mathrm{c}-\mathrm{h})$ and $0^{\circ}(\mathrm{i}-\mathrm{j})$, with respect to the crossed polarizers. Figure $\mathrm{f}$ ) is taken at the same voltage as figure e), but $60 \mathrm{~s}$ later. 


\section{WILEY-VCH}

(a)

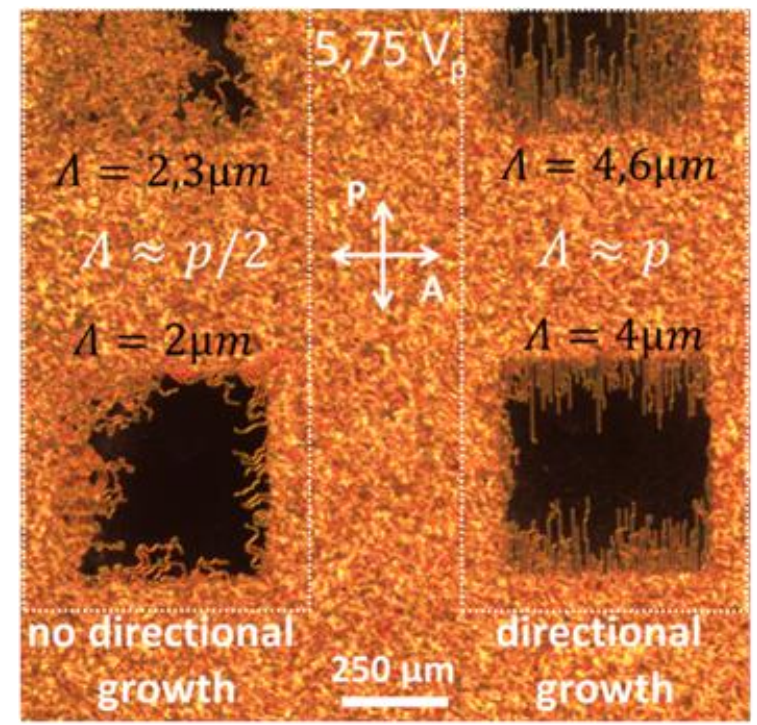

(b)

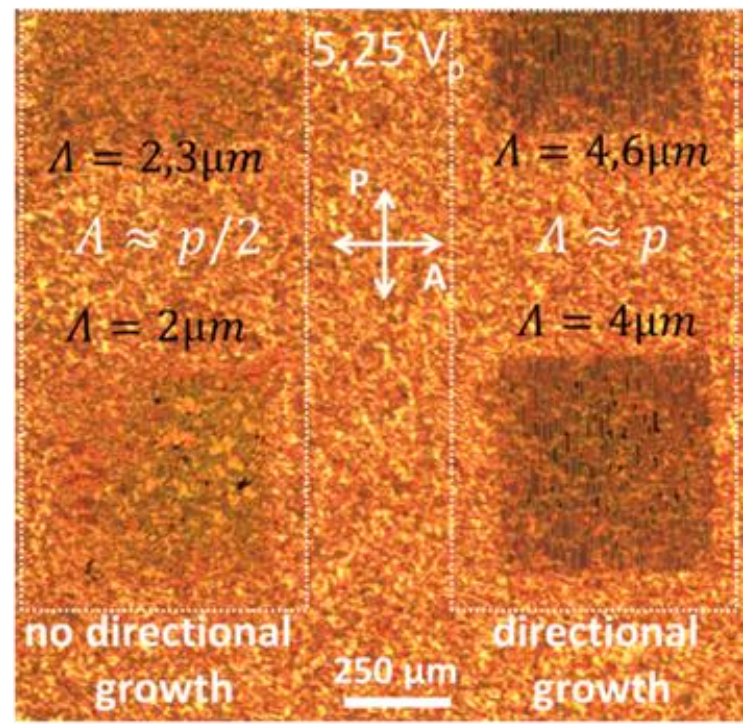

Figure 2. POM images at $5.75 \mathrm{~V}_{\mathrm{p}}$ (a) and $5.25 \mathrm{~V}_{\mathrm{p}}(\mathrm{b})$ for four different periodicities $\Lambda$ of the surface grating. Directional growth is only observed when the period $\Lambda$ is similar to the pitch p.
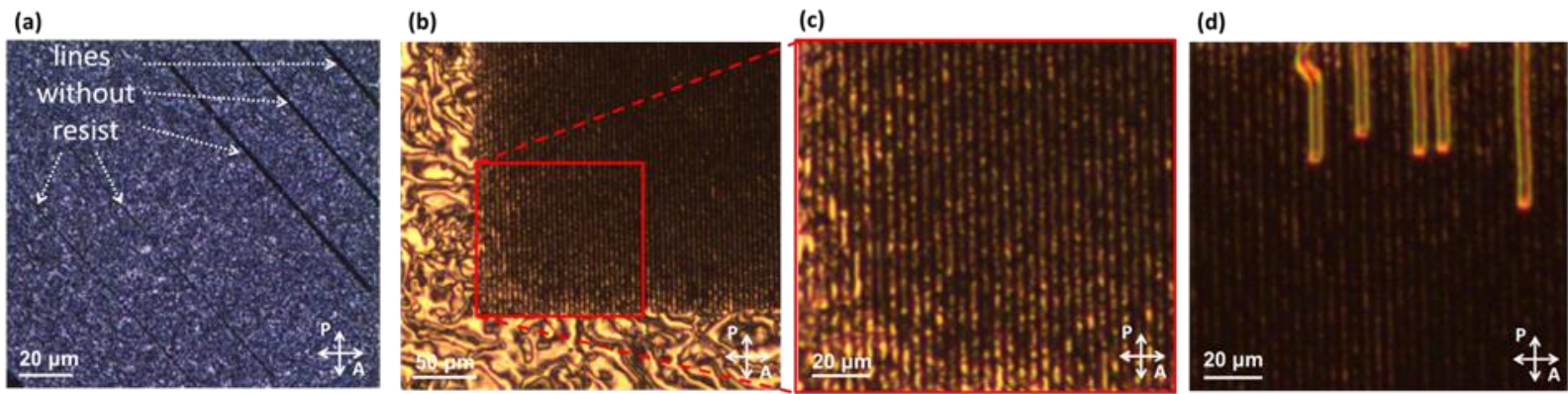

Figure 3. POM images at high voltage (above the dielectric unwinding threshold, when the director in the bulk is homeotropic) a) cell with isolated stripes where resist is removed (these stripes appear black, the smallest stripe has $2.3 \mu \mathrm{m}$ width); b,c) cell with a periodic grating structure; d) POM image of growing CFs in the grating area, when the voltage is reduced below the unwinding threshold.
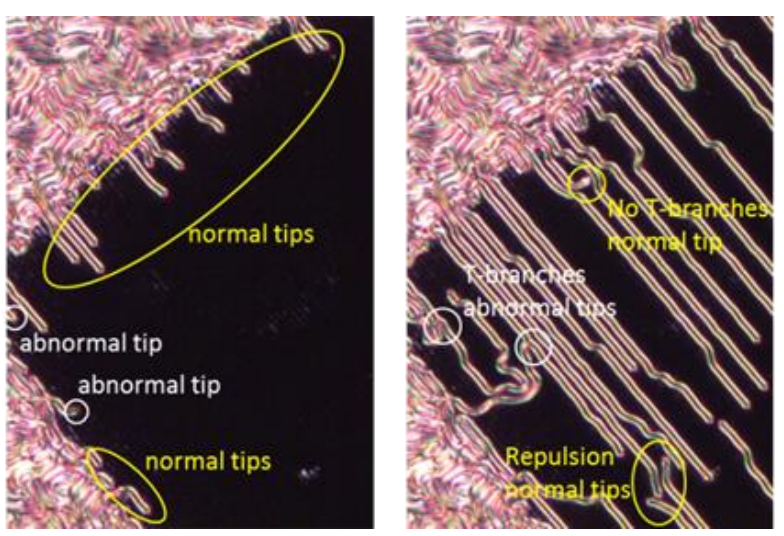

Figure 4. POM images at $5.75 \mathrm{~V}_{\mathrm{p}}$ (at different times) illustrating the dynamic growth of CFs and the interaction between different fingers. 


\section{WILEY-VCH}

(a)

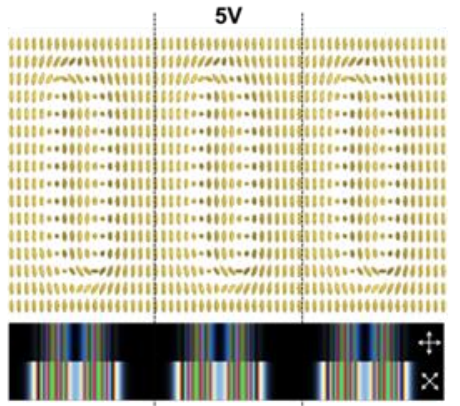

$\log _{10}\left|F_{\mathrm{e}}\right|\left[\mathrm{J} / \mathrm{m}^{2}\right]$

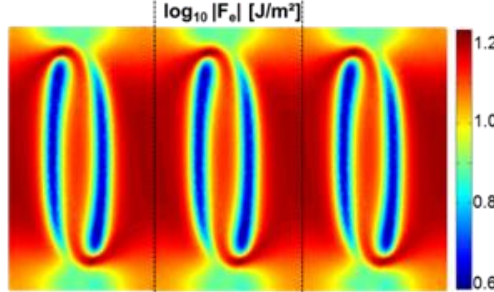

$\log _{10} F_{d}\left[J / m^{2}\right]$

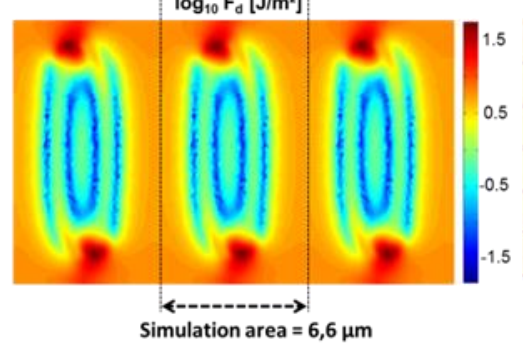

(b)

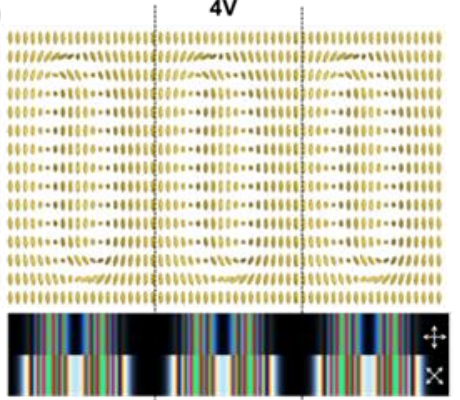

$\log _{10}\left|F_{\mathrm{e}}\right|\left[\mathrm{J} / \mathrm{m}^{2}\right]$

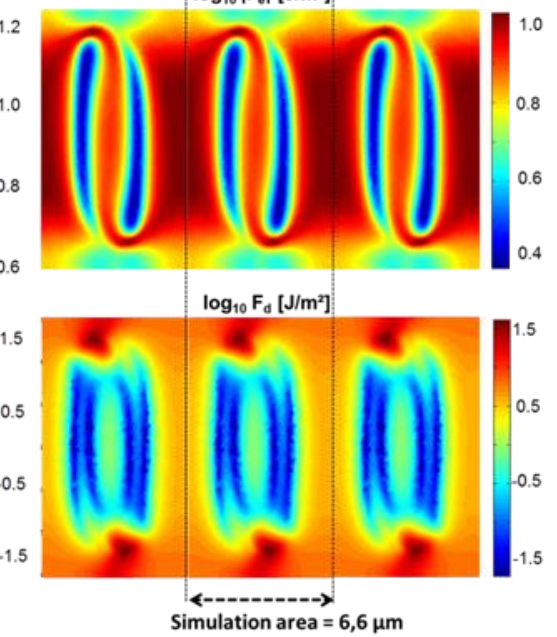

(c)
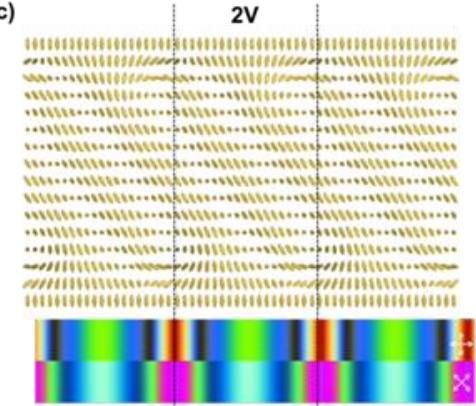

$\log _{10}\left|F_{e}\right|\left[J / m^{2}\right]$

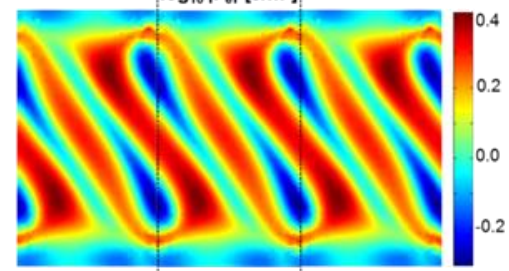

$\log _{10} F_{d}\left[J / m^{2}\right]$

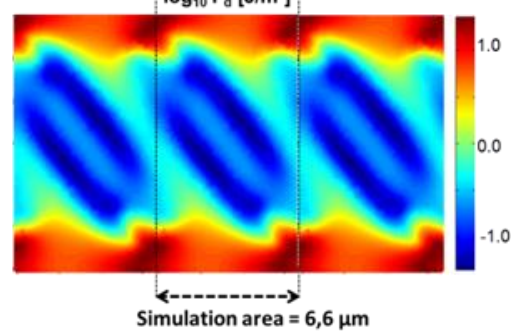

Figure 5. Simulated director configuration and transmission between crossed polarizers (top), electric energy density (middle) and distortion energy density (bottom) for a CF1 structure in a cell with homeotropic alignment layers at $5 \mathrm{~V}$ (a), 4V (b) and $2 \mathrm{~V} \mathrm{(c).} \mathrm{The} \mathrm{width} \mathrm{of} \mathrm{the}$ simulation area is $6.6 \mu \mathrm{m}$ and the cell thickness is $12 \mu \mathrm{m}$. Periodic boundary conditions are applied and 3 periods are shown. 
WILEY-VCH
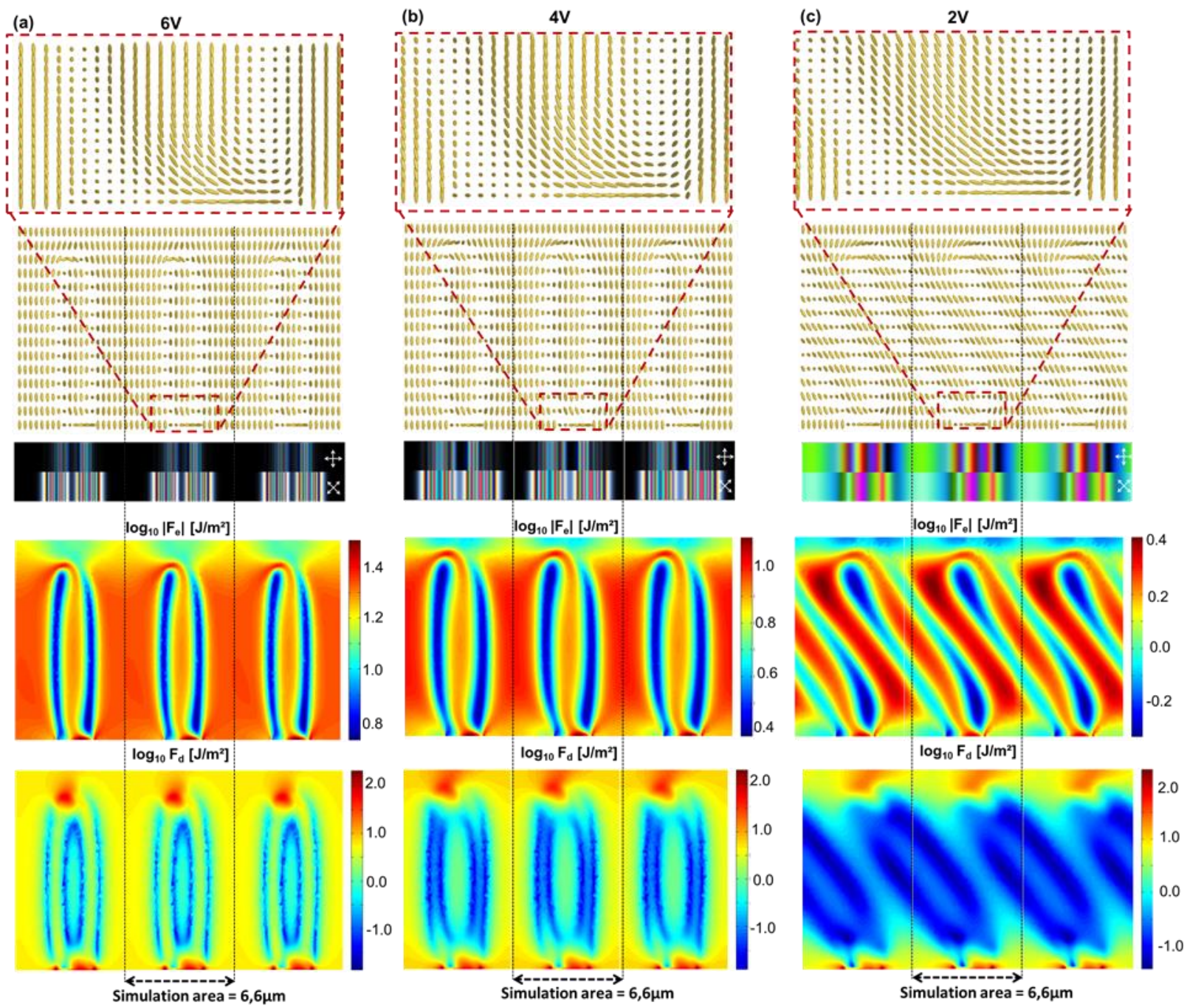

Figure 6. From top to bottom: simulated director configuration, with magnification of the region close to the bottom substrate; transmission between crossed polarizers; electric energy density; and distortion energy density, for a CF in a cell with homeotropic alignment at the top substrate and mixed homeotropic and planar alignment at the bottom substrate. Results at $6 \mathrm{~V}(\mathrm{a}), 4 \mathrm{~V}$ (b) and $2 \mathrm{~V} \mathrm{(c)} \mathrm{are} \mathrm{shown,} \mathrm{the} \mathrm{width} \mathrm{of} \mathrm{the} \mathrm{simulations} \mathrm{area} \mathrm{is} 6.6 \mu \mathrm{m}$ and the cell thickness is $12 \mu \mathrm{m}$. Periodic boundary conditions are applied and 3 periods are shown. The azimuthal anchoring strength in the planar alignment region is $10^{-5} \mathrm{~J} / \mathrm{m}^{2}$ (weak anchoring).

The table of contents entry should be 50-60 words long, and the first phrase should be bold. 


\section{WILEY-VCH}

E-beam lithography is used to pattern the surface anchoring and gain control over the liquid crystal alignment. By matching the period of the e-beam grating with the pitch of a chiral nematic liquid crystal, new chiral superstructures can be stabilized. The directional growth of the chiral superstructures along the grating stripes can be controlled by applying a voltage.

\section{Liquid crystal superstructures}

I. Nys, J. Beeckman*, K. Neyts

Surface mediated alignment of long pitch chiral nematic liquid crystal structures

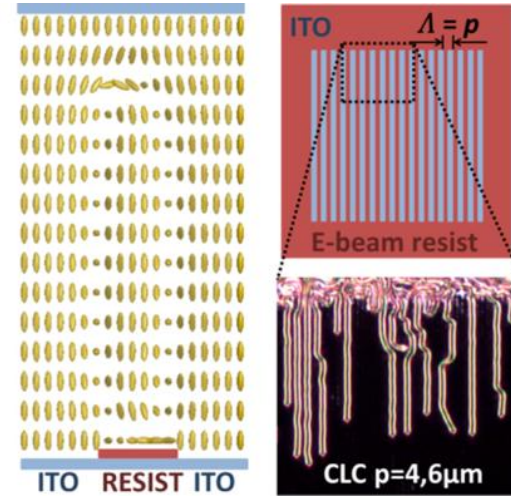




\section{WILEY-VCH}

Copyright WILEY-VCH Verlag GmbH \& Co. KGaA, 69469 Weinheim, Germany, 2016.

\section{Supporting Information}

\section{Surface mediated alignment of long pitch chiral nematic liquid crystal structures} Inge Nys, Jeroen Beeckman*, Kristiaan Neyts

\section{1) Details about the calculation of the transmission between crossed polarizers:}

The result of the Q-tensor simulation (on a triangular mesh) is interpolated onto a regular (x,z) grid and the azimuth and inclination of the director are calculated. The transmission for light propagating along the z-axis is calculated as a function of the x-coordinate, based on the Jones calculus, using the director values for the given $\mathrm{x}$-coordinate. Relatively rapid spatial variations of the director along $\mathrm{x}$ are present and diffraction and refraction may play a role in our cells, but are not taken into account. Therefore the simulated transmission results can only give an approximation for the transmission between crossed polarizers, which is more reliable when the variation along $\mathrm{x}$ is smaller. To obtain a rough color image, we combined the transmissions calculated for only three wavelengths in a color plot: $420 \mathrm{~nm}$ for blue, $520 \mathrm{~nm}$ for green and $620 \mathrm{~nm}$ for red (including more wavelengths in the spectrum may lead to more realistic results). The refractive indices of the $\mathrm{LC}$ that are used in the simulations are no=1.52 and ne $=1.74$.

\section{2) Additional simulation results:}

As explained in the manuscript, not every grating line is filled with a $\mathrm{CF}$ and the CFs are typically surrounded by a broader homeotropic unwound area. For this reason, we made the simulation domain a bit broader than the surface grating period $\Lambda$ (width $6.6 \mu \mathrm{m}$ instead of $\Lambda$ 


\section{WILEY-VCH}

$=4.6 \mu \mathrm{m}$ ) by increasing the width of the homeotropic areas while preserving the width of the planar region $(2.3 \mu \mathrm{m})$ (Figure 6 and 7 ). We also performed simulations with a width of 4.6 $\mu \mathrm{m}(2.3 \mu \mathrm{m}$ planar and $2.3 \mu \mathrm{m}$ homeotropic $)$ to show that this has only a minor influence on the results. The results are demonstrated in Figure $\mathbf{S 1}$ for different voltages. The azimuthal anchoring strength was assumed to be weak $\left(10^{-5} \mathrm{~J} / \mathrm{m}^{2}\right)$ to represent the experimentally observed planar degenerate anchoring, while the polar anchoring strength was set to $10^{-3} \mathrm{~J} / \mathrm{m}^{2}$. In this geometry, the CFs have less freedom to move laterally and therefore, the tilt of the cholesteric twist axis is less pronounced.

$6 \mathrm{~V}$
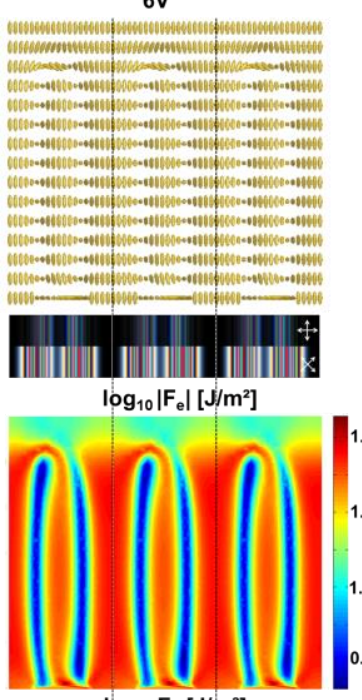

$\log _{10} F_{d}\left[J / m^{2}\right]$

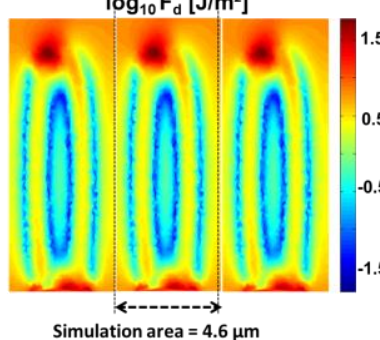

$4 \mathrm{~V}$
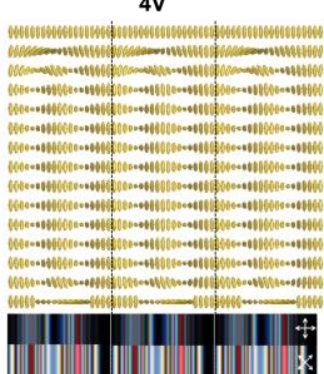

$\log _{10}\left|F_{e}\right|\left[J / m^{2}\right]$

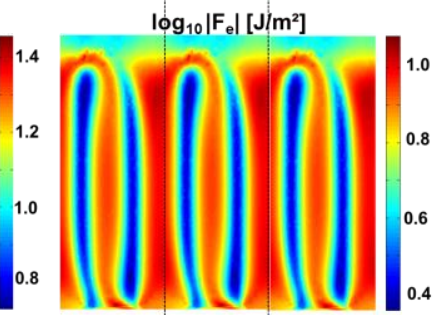

$\log _{10} F_{d}\left[J / m^{2}\right]$

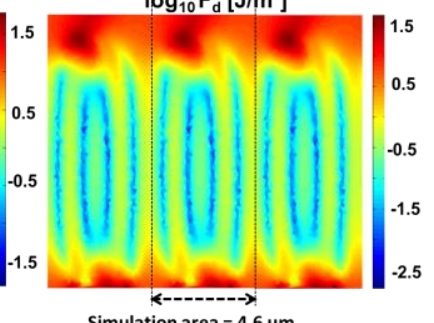

2V
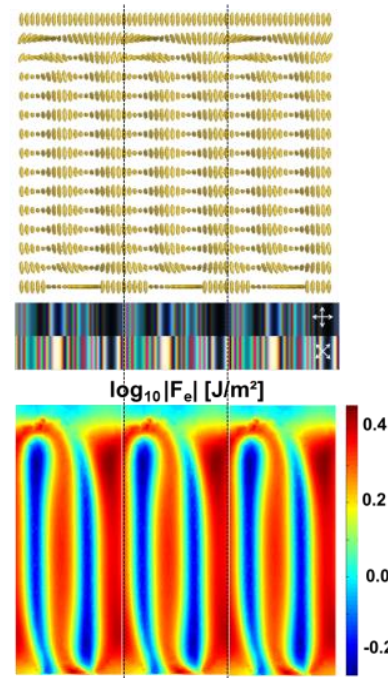

$\log _{10} F_{d}\left[J / m^{2}\right]$

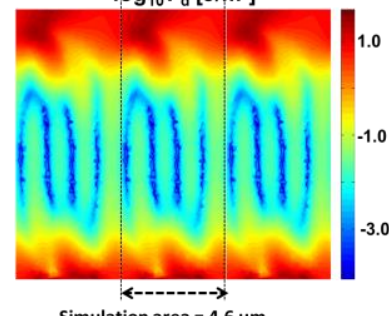

Simulation area $=4.6 \mu \mathrm{m}$
OV
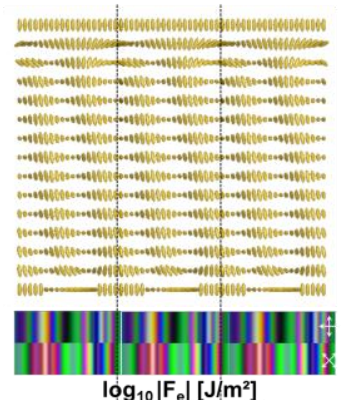

$\log _{10}\left|F_{e}\right|\left[\mathrm{J} / \mathrm{m}^{2}\right]$

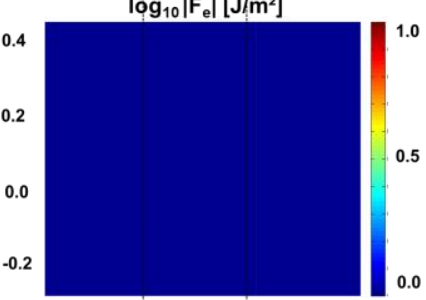

$\log _{10} F_{d}\left[J / m^{2}\right]$

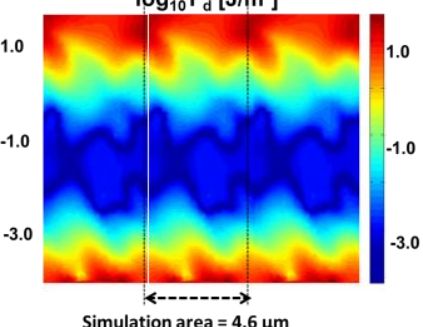

Figure S1. Simulated director configuration and transmission between crossed polarizers (top), electric energy density (middle) and distortion energy density (bottom) for a CF1 structure in a cell with homeotropic alignment at the top substrate and mixed planar homeotropic alignment at the bottom substrate. Results for 6V (a), 4V (b), 2V (c) and 0V (d) are shown. The width of the simulations area is $4.6 \mu \mathrm{m}$ and the cell thickness is $12 \mu \mathrm{m}$. Periodic boundary conditions are applied and 3 periods are shown.

Besides the simulations with weak azimuthal anchoring in the planar alignment region, also simulations with fixed planar (non-degenerate) anchoring were performed. In this case three 


\section{WILEY-VCH}

different azimuthal orientations of the planar anchoring were simulated and the alignment was assumed to be strong. As can be seen in Figure $\mathbf{S 2}$ this influences the director configuration in the region close to the patterned substrate. It is however clear that the alignment near the patterned substrate is only of minor importance for the overall cholesteric structure. For strong alignment along the x-axis (Figure S2c), the highest elastic energy is situated at the left side of the planar area, while this is at the right side for alignment along the y-axis. For planar alignment in the xy-direction de elastic energy density is more spread out over the whole area, which is similar for the case of weak azimuthal anchoring. By comparing the simulated results for the transmission between crossed polarizers (Figure 8 and Figure S3) with the experimental POM images (Figure 1) we can conclude that the simulations for weak azimuthal anchoring best resemble the experimental results.
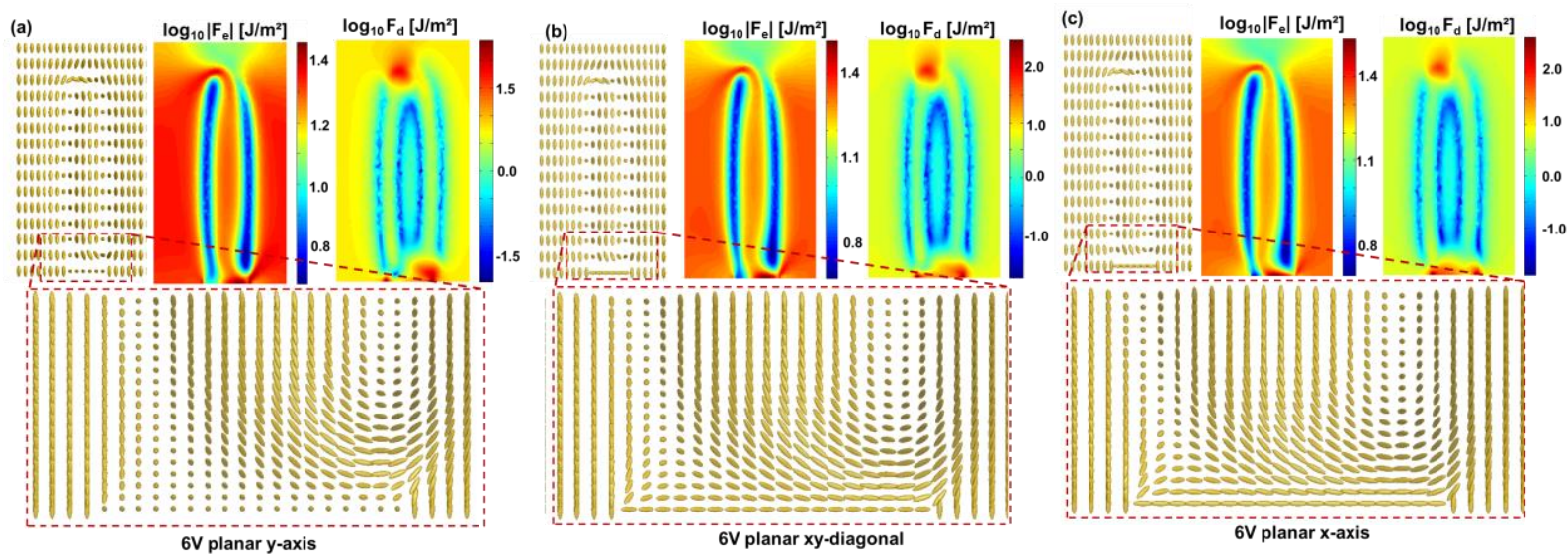

Figure S2. simulated director configuration, electric energy density and distortion energy density at $6 \mathrm{~V}$ for a cell with homeotropic alignment at the top substrate and mixed planar homeotropic alignment at the bottom substrate. The width of the simulations area is $6.6 \mu \mathrm{m}$ and the cell thickness is $12 \mu \mathrm{m}$. Strong planar alignment at the bottom substrate is assumed along the y-axis (a), along the xy-diagonal (b), or along the x-axis (c). 


\section{WILEY-VCH}
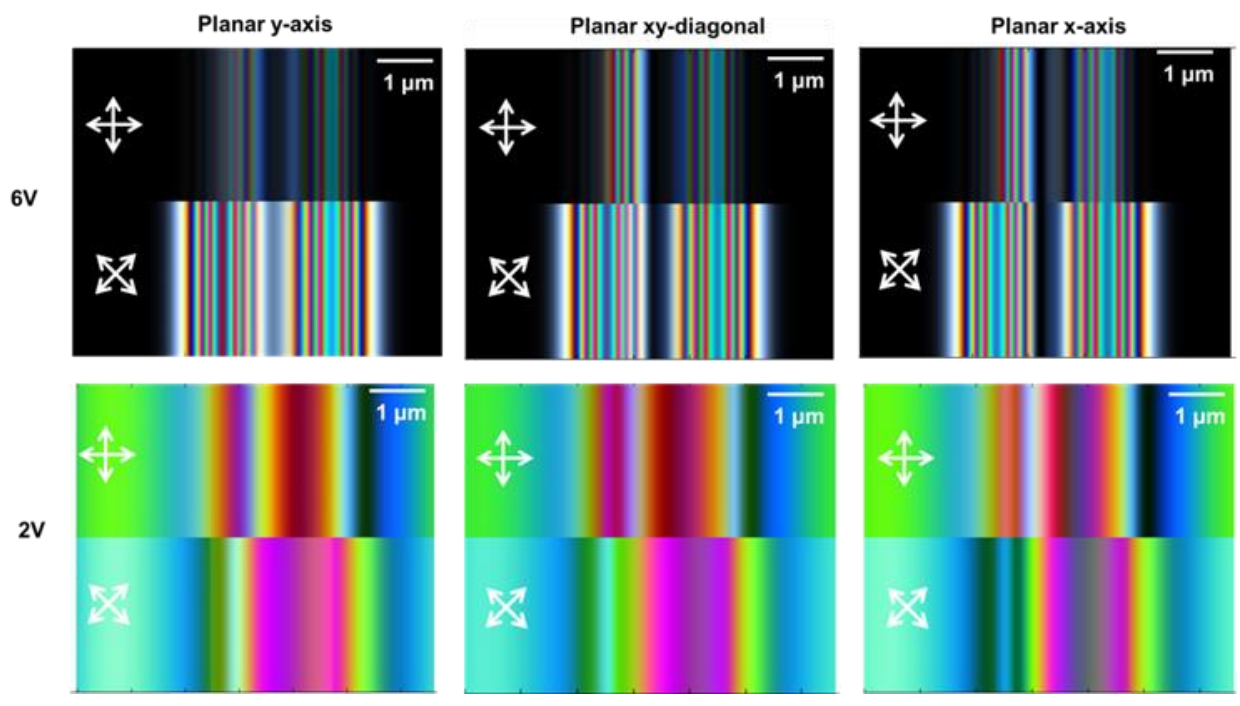

Figure S3. simulated transmission between crossed polarizers based on the Jones Calculus with three wavelengths for $6 \mathrm{~V}$ (top) and $2 \mathrm{~V}$ (bottom) for a cell with homeotropic alignment at the top substrate and stripes with (strong) planar and homeotropic alignment at the bottom substrate. The planar alignment is respectively along the $y$-axis (left), xy-diagonal (middle) and $\mathrm{x}$-axis (right).

\section{3) Additional experimental results:}

To further illustrate the dynamic growth of the CFs in our grating structure two movies are available online in the supporting information. The first movie (movie S1) shows that the nucleation and growth of CFs starts at the edges of the grating and illustrates the interaction of CFs propagating in opposite directions. In the beginning of movie S1 the voltage over the cell is decreased from $6 \mathrm{~V}_{\mathrm{p}}$ to $5.5 \mathrm{~V}_{\mathrm{p}}$. Two snapshots from the first movie are shown in figure 4 to summarize different types of interactions between CFs that are observed.

In the second movie (movie S2) the behavior of the CFs at slightly lower voltages is studied. In the beginning of movie $\mathrm{S} 2$ the voltage over the cell is decreased from $5.5 \mathrm{~V}_{\mathrm{p}}$ to $5 \mathrm{~V}_{\mathrm{p}}$. Even though the voltage is decreased in relatively small steps, the grating area never fills completely with parallel CFs and some homeotropically aligned areas are conserved. These unwound areas are less stable at lower voltages and the already present CFs start to buckle in the plane of the cell to fill the area. In our experiments, the anchoring of the CFs to the bottom substrate is not strong enough to prevent in-plane buckling and this mechanism dominates over nucleation and growth of new CFs at low voltages (Figure 1g, Movie S2). 